\title{
N-Butyl Cyanoacrylate Embolization in Management of latrogenic Renal Hemorrhages-Single-Center Study Evaluating Safety and Efficacy
}

\author{
Amey Narkhede ${ }^{1}$ Ajit Kumar Yadav ${ }^{1}$ Arun Gupta ${ }^{1}$ \\ ${ }^{1}$ Department of Interventional Radiology, Sir Ganga Ram Hospital, \\ New Delhi, India \\ J Clin Interv Radiol ISVIR 2022;6:75-82.
}

\begin{abstract}
Address for correspondence Arun Gupta, MD (Radiology), EBIR, Chairperson and Consultant, Department of Interventional Radiology, Department of Interventional Radiology (Room 20A), Sir Ganga Ram Hospital, Rajinder Nagar, Delhi- 60, India (e-mail: irdrarungupta@gmail.com).
\end{abstract}

\begin{abstract}
Objectives The aim of the study was to evaluate the efficacy of endovascular N-butyl cyanoacrylate (NBCA)/glue embolization alone in the management of iatrogenic renal injuries and its effect on renal function.

Methods Thirty-one patients who presented with significant iatrogenic renal injuries over a time period ranging from June 2013 to June 2018 were evaluated. The clinical features, lab parameters, and imaging findings were recorded. The embolizing agent used was exclusively NBCA. The success rate was calculated along with the effect of glue embolization on kidney in the form of postembolization ischemia and change in the serum creatinine levels in immediate pre- and postembolization period. The time between the iatrogenic injury and angioembolization as well as the time from angioembolization to discharge was also calculated. Recurrence was evaluated at 1 and 6 months follow-up.

Results NBCA embolization showed $100 \%$ technical and clinical success in our study and no recurrence was noted at follow-up. Glue was also able to achieve complete embolization in patients with coagulopathy $(n=14,45.1 \%)$. Percentage area deficit of normal renal blush in the postembolization check angiogram varied from 4 to $15.7 \%$

\section{Keywords}

- NBCA

- latrogenic renal injury

- renal artery pseudoaneurysm. with a mean deficit of $7.65 \%$. No significant difference in the serum creatinine was noted in immediate postembolization period.

Conclusions We conclude that glue is an effective embolizing agent for management of iatrogenic renal injuries even in coagulopathy patients with minimal area of parenchymal loss, no significant effect on renal functions, and no recurrence on 6 months follow-up.
\end{abstract}

\section{Introduction}

Endovascular approach of management has become the treatment of choice in the management of acute renal hemorrhages.
It is being employed as the first line of treatment in cases of deteriorating clinical status and renal function, massive bleed, or renal hemorrhage more than 72 hours. $^{1}$ published online September 20, 2021
DOI https://doi.org/

10.1055/s-0041-1732795. ISSN 2457-0214.
(C) 2021. Indian Society of Vascular and Interventional Radiology. All rights reserved.

This is an open access article published by Thieme under the terms of the Creative Commons Attribution-NonDerivative-NonCommercial-License, permitting copying and reproduction so long as the original work is given appropriate credit. Contents may not be used for commercial purposes, or adapted, remixed, transformed or built upon. (https://creativecommons.org/ licenses/by-nc-nd/4.0/)

Thieme Medical and Scientific Publishers Pvt. Ltd., A-12, 2nd Floor, Sector 2, Noida-201301 UP, India 
The most common embolic agent employed has been coils. ${ }^{2} \mathrm{~N}$-butyl cyanoacrylate (NBCA) or glue has been less commonly used, since coils are safe and easy to use and provide better control than glue unless there is coagulopathy or the embolization needs to be quick. Vascular injuries are seen in $15 \%$ of all renal procedures; however, the iatrogenic etiology accounts for $50 \%$ of renal injuries causing hemorrhage. ${ }^{3}$ Most of the vascular injuries show spontaneous resolution (70-90\%) with the remaining injuries requiring medical or interventional management. ${ }^{1}$

This study describes our experience of managing iatrogenic renovascular injuries exclusively with NBCA. Additionally, various factors involved in the management of iatrogenic renal bleed have been evaluated such as presence of coagulopathy.

The purpose of the study is to evaluate the efficacy as well as the renal adverse effects of exclusive usage of glue in embolization of iatrogenic causes of renal bleed based on our experience.

\section{Methods}

Since this was a retrospective observational study, Ethical Committee approval was waived off by the Institutional Review Board. The study included the patients referred to the department of interventional radiology in case of any clinical signs and symptoms of hemorrhage or fall in hemoglobin level following any diagnostic or therapeutic renal procedure. The patients having renal hemorrhage due to trauma or other noniatrogenic causes were excluded from this study. The patients were taken up for conventional angiography with embolization based on the computed tomography/ultrasonography (CT/USG) findings or on strong clinical suspicion when imaging findings were nondiagnostic. Postprocedure the patients' follow-up was recorded for signs of improvement based on clinical signs and symptoms and laboratory parameters. They were discharged after achieving stabilization. The patients were followed up at 1 and 6 months.

Our study collected and analyzed the data from June 2013 to June 2018 ( 5 years). It included a total of 31 patients in whom exclusively glue was used. The patients in this time period in whom other embolic materials were used or additional agents were used were excluded from this study. The patients' clinical data and imaging findings were obtained from picture archiving and communication system and medical records.

The dates of admission of patients, iatrogenic renal injury, angioembolization, and discharge were recorded. The time period between the iatrogenic injury and angioembolization as well as the time period from angioembolization to discharge in stable condition was calculated. The underlying renal disease of patient and the procedure leading to iatrogenic renal hemorrhage were noted. The clinical findings were also noted.

The laboratory parameters that were evaluated include fall in hemoglobin (in the time period from iatrogenic renal bleed and angioembolization), total leukocyte count (TLC), platelet count $(\mathrm{PC})$, and international normalized ratio (INR) values in the preembolization period. The effectiveness of glue embolization in coagulopathy with deranged INR and PC was evaluated. Coagulopathy has been defined as an INR > 1.5 and/or a $\mathrm{PC}<50 \times 10 / \mathrm{L}^{4}$

Numbers of units of blood transfused were recorded. The noninvasive imaging evaluations were done using USG and/or CT angiography. The conventional angiographic findings were noted that included pseudoaneurysm, arteriovenous fistula (AVF), and active hemorrhage. The kidney which was involved i.e. right or left or graft as well as the site of injury to the kidney i.e. upper/middle/lower pole were noted. Whether repeat embolization was required or not was also noted.

The causes of iatrogenic renal injuries consisted of biopsy (41.9\%; $n=13)$, partial nephrectomy $(9.6 \% ; n=3)$, percutaneous nephrolithotomy (PCNL) (35.4\%; $n=11$ ), PCNL with double J (DJ) stenting $(9.6 \% ; n=3)$, and DJ stenting $(3.2 \% ; n=1)$.

In patients with iatrogenic renal injury, age varied within 16 weeks to 80 years. Time from injury to arterial embolization varied from 0 to 31 days with mean duration of 9.58 days (standard deviation [SD]:10.7), whereas time from embolization to discharge varied from 0 to 40 days with a mean of 7.7 days (SD: 9.3). Mean fall of hemoglobin was $2.25 \mathrm{gm} / \mathrm{dL}$ with SD of $1.9 \mathrm{gm} / \mathrm{dL}$. There were two mean units of blood transfusion needed in patients with iatrogenic injury varying within 0 to 12 units transfusion. PC in patients was within 36 $\times 10^{9} / \mathrm{L}$ to $616 \times 10^{9} / \mathrm{L}$ range. INR in patients was within 0.9 to 1.54 range. The patients had mean TLC of $12.0 \times 10^{3} / \mathrm{dL}$ and were within 7.2 to $29.1 \times 10^{3} / \mathrm{dL}$ range.

Among the clinical symptoms, presence of hematuria, flank pain, and fever was noted. Seventy-four percent (23 patients) of our patients had flank pain. Fever was noted in $19 \%$ of patients ( 6 patients). Hematuria was most common presenting symptom (74\%) with $61 \%$ of patient showing single episode of hematuria (19 patients) and 12\% of patients showing recurrent episode of hematuria (4 patients). Nineteen patients showed presence of both hematuria and flank pain as a presenting symptom. However, four patients had neither hematuria nor flank pain. These four patients were suspected to have renal injury because of significant fall in hemoglobin and its related symptoms (fall in blood pressure and tachycardia reduction in oxygen saturation).

Finally, the technical and clinical success rates were calculated to assess the efficacy of our procedure. The effect of embolization on vascular supply to the kidney was assessed by evaluating area of absent blush (infarction) postembolization. Effect on renal function was noted by observing any significant change in value of serum creatinine post procedure.

The technical success was defined as total occlusion of target vessels and resolution of angiographic abnormality (pseudoaneurysm/AVF/active bleed). Clinical success was defined as resolution of the clinical signs and symptoms with discharge in stable condition and no recurrence of bleed on follow-up. Follow-up was done after 1 and 6 months of discharge. 


\begin{tabular}{|c|c|c|c|c|c|c|c|c|c|c|c|c|c|c|c|c|c|c|c|c|c|c|c|c|}
\hline 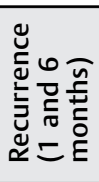 & 2 & z & 2 & 2 & 2 & z & 2 & 2 & z & 2 & z & z & 2 & 2 & 2 & z & \begin{tabular}{l|l}
2 & $\frac{5}{2}$
\end{tabular} & \begin{tabular}{l|l}
$z$ & \\
$z$
\end{tabular} & $\stackrel{2}{z}$ & \begin{tabular}{l|l|l|l}
2 & $\frac{5}{2}$
\end{tabular} & z & z & z & z \\
\hline 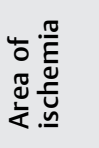 & 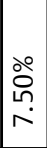 & $\begin{array}{l}\text { ○ें } \\
\text { ஸ่ } \\
\text { ஸे }\end{array}$ & 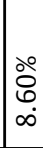 & $\begin{array}{l}\stackrel{\circ}{\circ} \\
\text { m. } \\
\overline{1}\end{array}$ & ○े & $\begin{array}{l}\stackrel{\circ}{\circ} \\
\infty \\
\infty\end{array}$ & 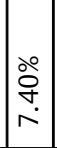 & $\begin{array}{l}\stackrel{\circ}{0} \\
\dot{0} \\
\dot{+}\end{array}$ & $\begin{array}{l}\text { oे } \\
\text { ñ. } \\
\text { ò }\end{array}$ & $\stackrel{\text { वे }}{ }$ & 总 & $\mid$ & 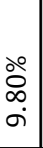 & $\stackrel{一}{\circ}$ & 品 & 总 & 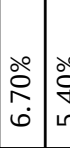 & 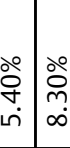 & 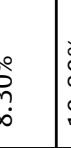 & 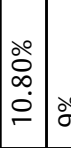 & & 总 & 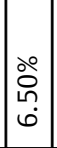 & ه্ণ \\
\hline 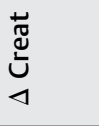 & 0 & $\hat{m}$ & $\stackrel{\infty}{0}$ & 0 & 0 & na & 0 & 0 & on. & ${ }_{0}^{m}$ & 0 & 0 & $\begin{array}{l}9 \\
\dot{i} \\
1\end{array}$ & 0 & $\bar{\varphi}$ & - & $\begin{array}{l}\hat{0} \\
\end{array}$ & 0 & b & $\left.\mid \begin{array}{l}0 \\
0 \\
0 \\
1\end{array}\right]$ & 0 & $\stackrel{t}{0}$ & $\stackrel{m}{o}$ & 0 \\
\hline 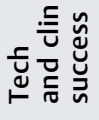 & 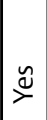 & $\stackrel{\check{\varpi}}{\rightleftharpoons}$ & $\stackrel{\circlearrowright}{\rightleftharpoons}$ & 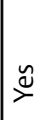 & $\stackrel{\tilde{u}}{>}$ & $\stackrel{\check{\nu}}{\rightleftharpoons}$ & $\stackrel{\tilde{u}}{\rightleftharpoons}$ & 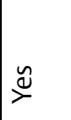 & $\stackrel{\check{\nu}}{\rightleftharpoons}$ & $\stackrel{\check{\Perp}}{\rightleftharpoons}$ & $\stackrel{\check{u}}{\rightleftharpoons}$ & $\stackrel{\stackrel{u}{\nu}}{\rightleftharpoons}$ & $\stackrel{\tilde{u}}{\succ}$ & $\stackrel{\check{u}}{\succ}$ & 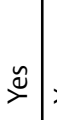 & $\stackrel{\check{\nu}}{\succ}$ & $\stackrel{\check{U}}{\rightleftharpoons}$ & 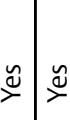 & E & 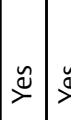 & $\stackrel{\tilde{\nu}}{\check{\nu}}$ & 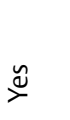 & 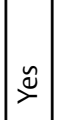 & 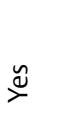 \\
\hline $\begin{array}{l}\text { पू } \\
\text { É } \\
\text { Eे } \\
\text { W }\end{array}$ & $\frac{g}{v}$ & $\frac{0}{v}$ & $\frac{g}{U}$ & $\frac{\partial}{v}$ & $\frac{g}{\Xi}$ & $\frac{\mathrm{g}}{\mathrm{U}}$ & $\frac{g}{\Xi}$ & $\frac{\mathrm{g}}{\mathrm{U}}$ & $\frac{\stackrel{D}{v}}{v}$ & $\frac{g}{3}$ & $\frac{\Xi}{v}$ & $\frac{g}{v}$ & $\frac{\mathrm{g}}{\mathrm{v}}$ & $\frac{\Xi}{v}$ & $\frac{\mathscr{g}}{\mathrm{J}}$ & $\frac{\frac{g}{v}}{\mathrm{v}}$ & $\frac{g}{U}$ & \begin{tabular}{l|l}
$\frac{g}{v}$ & $\frac{g}{v}$ \\
$v$
\end{tabular} & $\frac{5}{5}$ & $\frac{\Xi}{v}=\frac{9}{\mathcal{U}}$ & $\frac{\frac{g}{v}}{v}$ & $\frac{\stackrel{g}{U}}{v}$ & $\frac{g}{\mathrm{~g}}$ & $\frac{\stackrel{\Xi}{v}}{v}$ \\
\hline 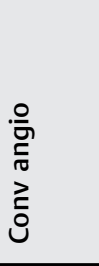 & 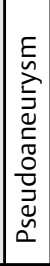 & 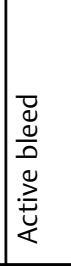 & 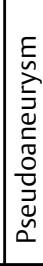 & 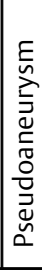 & 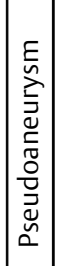 & 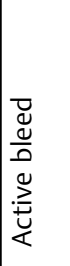 & 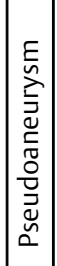 & 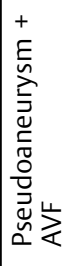 & 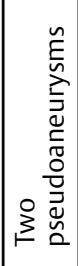 & 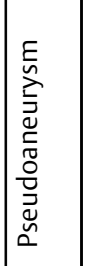 & 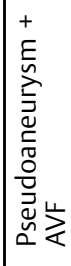 & 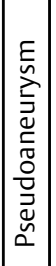 & 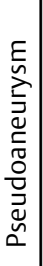 & 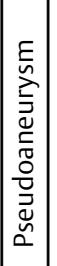 & 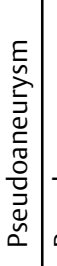 & 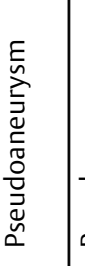 & 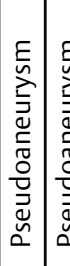 & 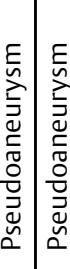 & 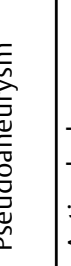 & 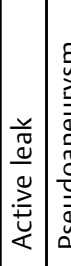 & 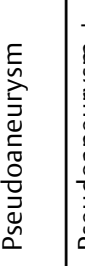 & 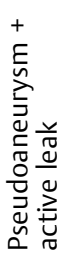 & 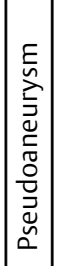 & 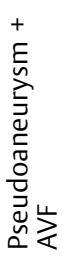 \\
\hline$\underline{\underline{\alpha}}$ & بִ & $\stackrel{\sim}{\sim}$ & - & $\stackrel{\sim}{\sim}$ & 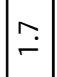 & - & $\stackrel{0}{-}$ & $\cong$ & $\stackrel{0}{-}$ & $\stackrel{-}{\sim}$ & g̊. & 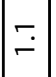 & $\stackrel{g}{0}$ & $=$ & $=$. & - & -2 & $\stackrel{-}{\rightleftharpoons}$ & $\underline{v}$ & $=$ & - & $\stackrel{\text { 무 }}{\longrightarrow}$ & 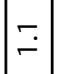 & $\stackrel{\sim}{\longrightarrow}$ \\
\hline 롱 & $\stackrel{0}{m}$ & $\mathscr{F}$ & $\stackrel{\operatorname{nn}}{\sim}$ & $\stackrel{\infty}{\circ}$ & 욤 & $\stackrel{\infty}{+}$ & ষ্লি & $\stackrel{\infty}{\stackrel{\infty}{N}}$ & ฮิ & $\stackrel{\infty}{\sim}$ & $\stackrel{\aleph}{N}$ & $\frac{6}{6}$ & ஜ & 文 & $\tilde{n}$ & ஓ & 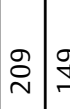 & $\stackrel{g}{\stackrel{g}{\sim}} \underset{\sim}{\approx}$ & vे & in & : & $\stackrel{\infty}{+}$ & $\stackrel{\bar{\infty}}{\sim}$ & $\stackrel{\infty}{\stackrel{\infty}{N}}$ \\
\hline$\sum_{\substack{\underline{n} \\
\frac{n}{n}}}$ & 0 & in & $\sim$ & - & $\sim$ & $\sim$ & 0 & 0 & $\sim$ & $\theta$ & 0 & 0 & - & 0 & $\sim$ & $m$ & $\nabla 0$ & 0 o $1-$ & - & 6 & $\sim$ & $\simeq$ & - & $m$ \\
\hline $\begin{array}{l}. \subseteq \\
\overline{\bar{\varpi}} \\
\text { 모 }\end{array}$ & $F$ & $\bar{\varphi}$ & $\bar{i}$ & $m$ & $\stackrel{m}{\sim}$ & $\bar{f}$ & 0 & 0 & $\stackrel{\infty}{\underset{\sim}{+}}$ & 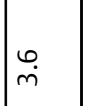 & 0 & 0 & 足 & 0 & 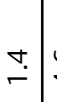 & $\begin{array}{l}\dot{\varphi} \\
\dot{\sim}\end{array}$ & $\hat{i}$ & 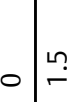 & $?$ & $\bar{i} \mid \bar{r}$ & $\hat{m}$ & $m$ & $\mid \begin{array}{l}\infty \\
0 \\
0\end{array}$ & in \\
\hline 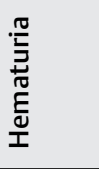 & $\frac{0}{2}$ & $\frac{0}{2}$ & z & 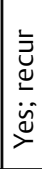 & $\stackrel{\tilde{u}}{\rightleftharpoons}$ & 2 & $\stackrel{\tilde{u}}{\rightleftharpoons}$ & $\stackrel{\ddot{\nu}}{\nu}$ & $\stackrel{\check{\nu}}{\succ}$ & $\stackrel{\check{\nu}}{\rightleftharpoons}$ & 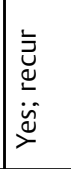 & 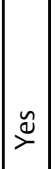 & $\stackrel{\tilde{u}}{\check{\nu}}$ & $\stackrel{\stackrel{\Perp}{\rightleftharpoons}}{\rightleftharpoons}$ & $\stackrel{\tilde{\nu}}{\nu}$ & $\stackrel{\check{\nu}}{\check{\nu}}$ & 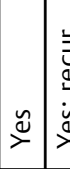 & 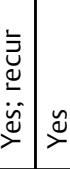 & 气̃ & 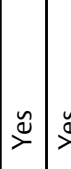 & $\stackrel{\check{\nu}}{\check{\nu}}$ & $\stackrel{\tilde{\nu}}{\nu}$ & $\stackrel{\check{y}}{\rightleftharpoons}$ & 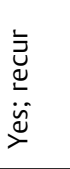 \\
\hline 旁 & $\stackrel{\tilde{y}}{>}$ & z & $\stackrel{\check{\nu}}{\nu}$ & $\stackrel{\check{\nu}}{\nu}$ & $\stackrel{\tilde{y}}{>}$ & z & $\stackrel{\tilde{y}}{>}$ & $\stackrel{\check{\nu}}{\rightleftharpoons}$ & 2 & $\stackrel{\check{\nu}}{\rightleftharpoons}$ & $\stackrel{\check{\nu}}{\varnothing}$ & 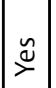 & $\stackrel{\check{\Perp}}{\succ}$ & $\stackrel{\check{\Perp}}{\succ}$ & $\stackrel{0}{z}$ & $\stackrel{\check{\Perp}}{\succ}$ & 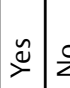 & 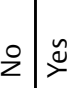 & y & 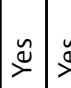 & $\stackrel{\check{\Perp}}{\rightleftharpoons}$ & $\stackrel{\check{y}}{>}$ & zo & $\stackrel{\check{y}}{>}$ \\
\hline 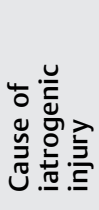 & 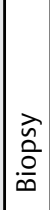 & 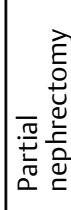 & $\begin{array}{l}\grave{\hat{~}} \\
\stackrel{0}{\infty}\end{array}$ & 䓀 & $\sum_{0}$ & 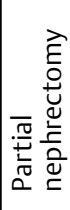 & $\underset{\Sigma}{z}$ & $\sum_{a}$ & $\begin{array}{l}\varkappa \\
\stackrel{\hat{o}}{0} \\
\frac{0}{\infty}\end{array}$ & 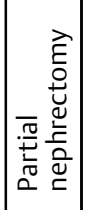 & 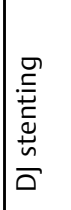 & 竎 & 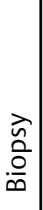 & 剖 & $\begin{array}{l}\overline{\hat{u}} \\
\stackrel{0}{\infty}\end{array}$ & 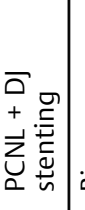 & 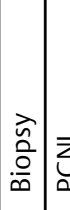 & 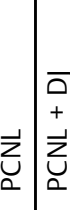 & 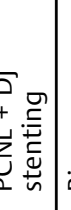 & 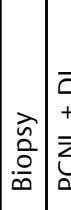 & 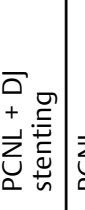 & $\sum_{\text {Ż }}$ & $\sum_{0}$ & $\underbrace{}_{\text {Ż }}$ \\
\hline 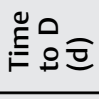 & 0 & $\stackrel{N}{N}$ & $\sim$ & - & - & - & $m$ & $N$ & $=$ & in & in & $\nabla$ & $\sim$ & $\sim$ & $\sim$ & เn & in 7 & 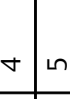 & ก & $\neq 1$ & $m$ & $\stackrel{\nu}{N}$ & $\nexists$ & $m$ \\
\hline 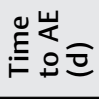 & 0 & 0 & 0 & $\bar{m}$ & $\bar{\sim}$ & in & 0 & 6 & in & $\sim$ & $\simeq$ & 0 & $\bar{m}$ & - & m & $\stackrel{\llcorner}{\leftarrow}$ & $\ln \mid \stackrel{m}{n}$ & $\stackrel{\sim}{\sim} \lesssim$ & v & $m \stackrel{n}{n}$ & $\stackrel{\llcorner}{\sim}$ & - & $\stackrel{\infty}{\sim}$ & 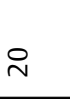 \\
\hline 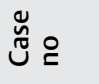 & - & $\sim$ & $m$ & $\theta$ & 乞n & | & r & $\infty$ & $a$ & $\circ$ & $\mp$ & $\simeq$ & $m$ & 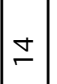 & $\stackrel{n}{\llcorner}$ & $\stackrel{\varphi}{\circ}$ & $\neq 1$ & $\stackrel{\infty}{\square}$ & T & $\stackrel{\sim}{2}$ & $\bar{\nu}$ & $\approx$ & $\cong$ & $\stackrel{\Delta}{\sim}$ \\
\hline
\end{tabular}



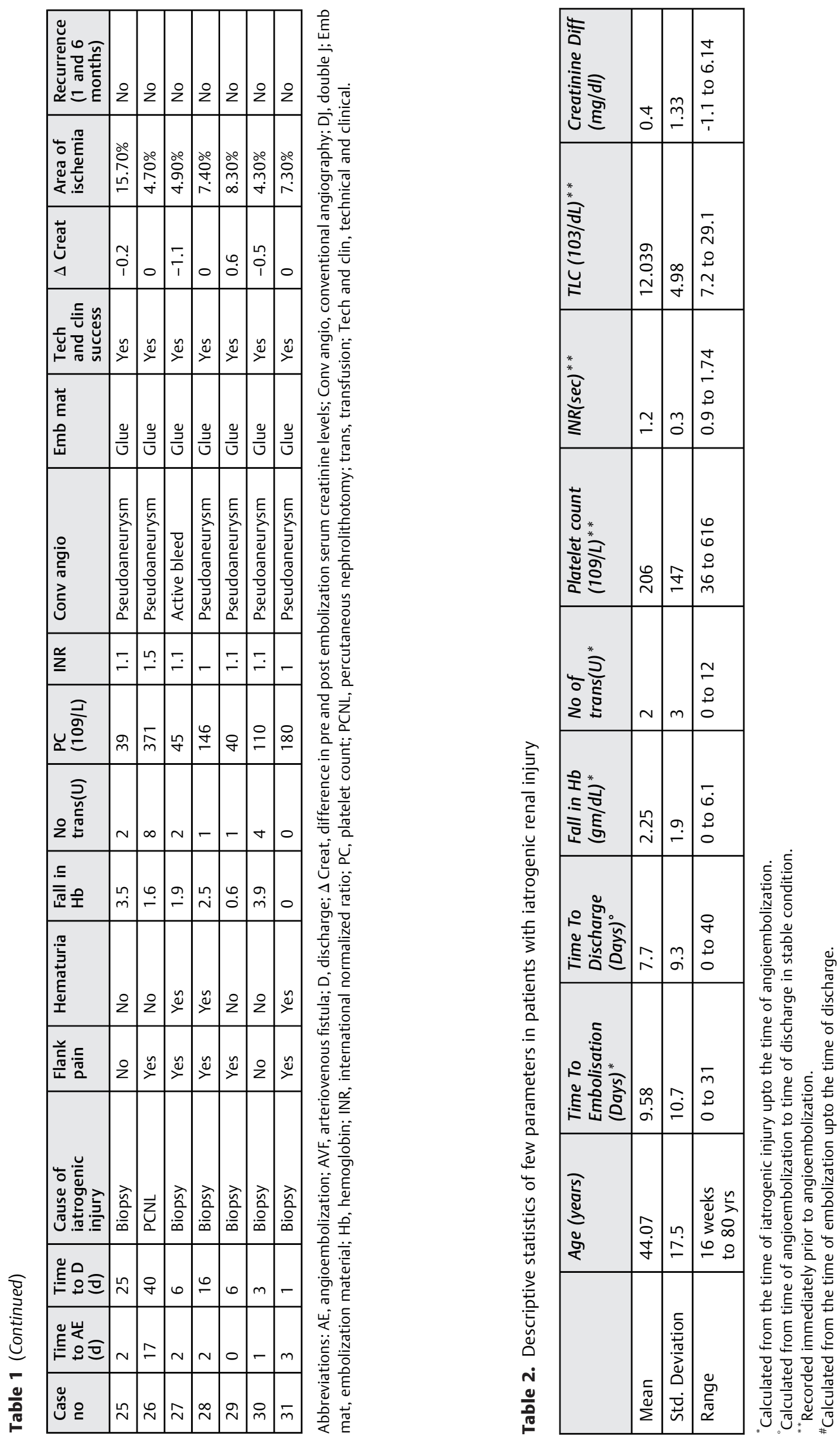
The postembolization infarcted area was assessed immediately on postembolization check angiography in the cath lab. The maximum circumference of the area showing $n$ blush was traced to calculate the approximate area of infarction on the two-dimensional image. Additionally, the difference in serum creatinine levels before and after the procedure was also assessed to evaluate the effect of embolization on renal function (-Tables 1 and $\mathbf{2}$ ).

\section{Technique}

The procedure was performed by two experienced interventional radiologists with 10 to 15 years of experience. The right common femoral artery was catheterized using a $5 \mathrm{Fr}$ sheath (Trans-Intro, Translumina Therapeutics, Dehradun, Uttarakhand, India). Right or left main renal artery was accessed using a cobra catheter (Cook Medical, Indiana, United States) and hydrophilic guide wire (Radifocus, Terumo, Tokyo, Japan). Renal angiogram was taken to establish or to confirm the diagnosis; super selective cannulation of the target vessel was done using 2.7 Fr micro catheter system (Progreat $\alpha$, Terumo, Tokyo, Japan). The microcatheter was flushed with $5 \%$ dextrose to prevent glue polymerization into the microcatheter itself. Then glue was injected into the target site in a ratio ranging from 1:1 to 1:4 (NBCA: Lipiodol).

The dilution of glue was based upon the distance of microcatheter tip from the target site. Immediately after achieving glue embolization at the target site the microcatheter was removed in single quick motion. Finally, a check renal angiogram was taken to confirm complete and successful embolization.

\section{Results}

The total numbers of patients with coagulopathy were 14 (45.1\%). Reduced PC was noted in seven patients (22.5\%). Glue used in these patients as an embolizing agent showed $100 \%$ of technical and clinical success rate. Raised INR value was noted in nine patients (29.03\%). These patients also showed $100 \%$ technical and clinical success with glue embolization. Among these patients of coagulopathy mentioned above, there were two patients $(6.45 \%)$ who had deranged INR and PC both.

Follow-up of the patients at 1 and 6 months showed no evidence of recurrence. Hence, it was observed from our study that glue acts as a successful embolizing agent in patients with coagulopathy.

Noninvasive diagnostic measures were indicative of renal hemorrhage in 22 cases. Out of these, 10 patients underwent CT angiography and 12 patients underwent USG screening prior to taking up the patient for conventional angiography. The exact cause of hemorrhage (pseudoaneurysm, AVF, and extravasation) could be detected in 10 cases that underwent $\mathrm{CT}$ angiography. In rest of the 12 cases, USG showed presence of only perinephric hematoma and exact cause could not be delineated. The remaining nine cases showed significant signs and symptoms of hemorrhage in the postprocedure period, and hence, on the basis of high clinical suspicion, they were directly taken up for conventional angiography with embolization.

The most common conventional angiographic finding was pseudoaneurysm in 27 patients (87.1\%). Of these, three patients showed simultaneous presence of AVF. One of the patients showed presence of two pseudoaneurysms. One of the patients showed presence of pseudoaneurysm with active bleed. Only active bleed was noted in 4/31 patients (12.9\%).

The percentage deficit that is the approximate area of infarction varied from a range of 4 to $15.7 \%$ with a mean deficit of $7.65 \%$ in renal parenchymal blush post glue embolization. This amount of blush deficit led to no significant difference in renal functions.

After embolization, 15 patients had no fall or rise in creatinine postprocedure. Eleven patents showed a fall in creatinine level ranging from 6.14 to $0.22 \mathrm{mg} / \mathrm{dL}$ with a mean fall of $1.32 \mathrm{mg} / \mathrm{dL}$. Five patients showed a rise in creatinine level ranging from 0.15 to $1.1 \mathrm{mg} / \mathrm{dL}$ with a mean rise of 0.64 $\mathrm{mg} / \mathrm{dL}$. Hence, no significant change in creatinine levels was noted.

Repeat embolization was not required in any of these patients in the next 24 hours. We achieved complete technical and clinical success in all of our 31 patients with glue embolization. The patients were called for 1 and 6 months follow-up. None of the patients showed signs and symptoms of recurrence on clinical evaluation.

Three of the cases have been shown and described in -Figs. 1-3.

\section{Discussion}

Endovascular management has been established as the standard of treatment for renal hemorrhages. It offers advantages of being minimally invasive with superselective embolization of the area of bleeding with minimal damage to normal renal parenchyma thus precluding the need of surgery (nephrectomy). ${ }^{5}$

Various embolic agents have been described in literature in the management of renal bleed. The choice depends upon the vascular anatomy and the pathology involved and majorly on the preference of interventional radiologist. Review of literature shows that metallic coils have been the preferred embolic agents by various authors owing to their controlled deploy ability. However, coil embolization is associated with the complication of coil migration and occlusion of nontarget vessels. $^{6}$

Although NBCA is also reported to result in nontarget embolization specially in inexperienced hands, it is a rather a cost-effective embolizing agent. The first report on use of glue was published in 1975 in the treatment of gastrointestinal bleed. ${ }^{7}$ Since then, there has been a rise in its use.

Achievement of embolization by coil requires the patients' coagulation parameters to be normal range. In cases of low PC, platelet dysfunction, deranged INR, and abnormal clotting factors, incomplete and delayed embolization can occur. $^{8}$ 


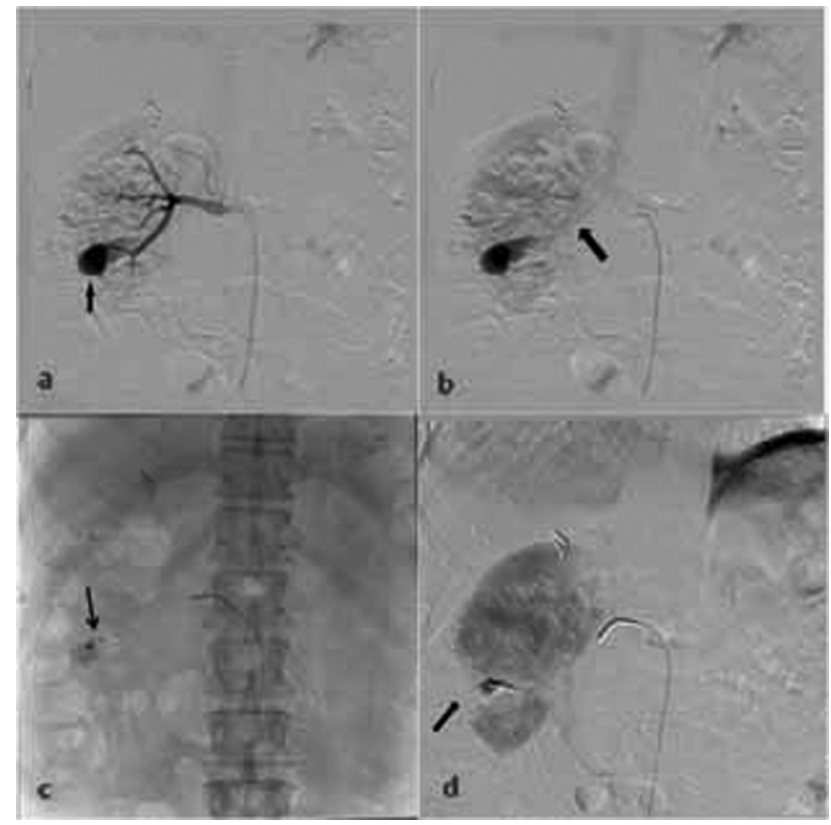

Fig. 1 (Case No. 24 in Table 1). A 62-year-old male who underwent percutaneous nephrolithotomy for right-sided renal calculi with postprocedure right-sided flank pain and hematuria.(A) The angiographic image shows that a pseudoaneurysm (arrow) is noted arising from a branch of right lower pole renal artery. (B) Early filling of the right main renal vein is noted (arrow) suggesting the simultaneous presence of an arteriovenous fistula.(C) Microcatheter is progressed up to the neck of pseudoaneurysm and $50 \%$ glue is injected into the pseudoaneurysm to fill it up and also the supplying artery (arrow). (D) Postembolization check angiogram shows parenchymal blush deficit $(\sim 4.20 \%)$ (arrow) in the region of embolization with nonfilling up of the pseudoaneurysm and arteriovenous fistula.

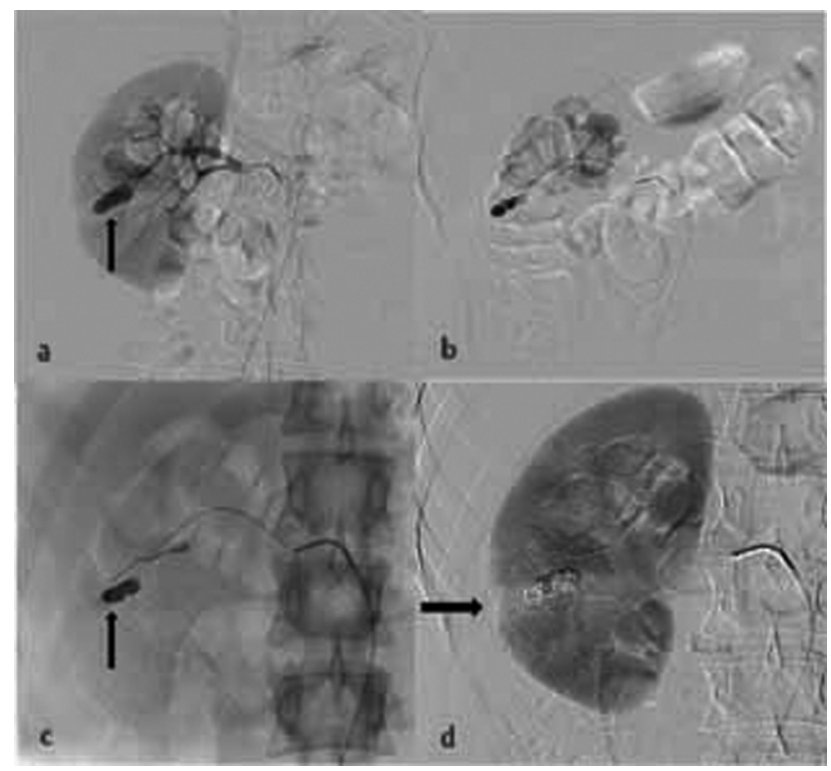

Fig. 2 (Case No. 19 in Table 1). A 29-year-old male with right renal calculi, status post right percutaneous nephrolithotomy and double J stenting with significant hematuria. (A) The angiogram shows a pseudoaneurysm arising from a branch of right middle pole renal artery (arrow). (B) The microcatheter is traversed up to the pseudoaneurysm neck and contrast in injected slowly to confirm the catheter position. (C) Complete glue embolization of the pseudoaneurysm is achieved (arrow) with minimal leak into the pelvicalyceal system. (D) Postembolization check angiogram.

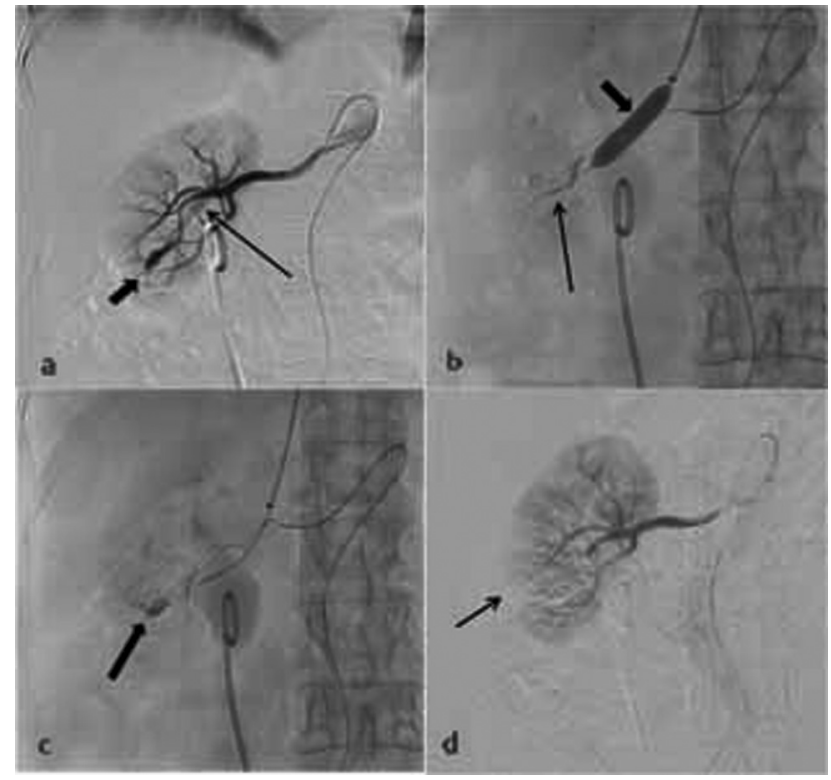

Fig. 3 (Case No. 11 in Table 1). A 80-year-old female, status post right sided double J stenting having right-sided flank pain with repeated episodes of hematuria. (A) Renal angiogram shows a pseudoaneurysm arising from a branch of right lower pole renal artery (short thick arrow). Additionally, very early filling of the right main renal vein is noted (long thin arrow) indicating this to be a high flow arteriovenous fistula. (B) Short thick arrow shows the balloon in the inflated state deployed in the right main renal vein. Long thin arrow shows the glue that is being injected via the microcatheter present in the arterial system. (C) Glue cast (arrow) is noted occupying the pseudoaneurysm and the proximal supplying arteries. The balloon is in deflated state. (D) Postembolization check angiogram with parenchymal area deficit of $\sim 6.20 \%$ (arrow; ). Causes of iatrogenic renal injury, time to angioembolization, time to discharge, clinical and lab parameters, angiographic imaging findings, embolization material used, technical and clinical success, effect on serum creatinine, area of parenchymal deficit postembolization, and recurrence at 1 and 6 months follow-up.

Glue embolization is independent of these factors. ${ }^{8}$ Kim et al have also reported a clinical success of $87.5 \%$ in 14 patients using NBCA as the only embolic agent (64\%) or in combination with other embolic agent (36\%). ${ }^{9}$ The polymerization of NBCA occurs immediately on contact with anionic blood. ${ }^{10-12}$ The various mechanisms causing the immediate embolization of blood vessels include glue cast and thrombus formation, adhesion of the glue to the inner vascular wall, and vascular endothelial wall damage causing acute necrotizing vasculitis, eventually leading to scarring and fibrosis of the vessel. ${ }^{13}$ Glue embolization is instant, permanent, and causes complete vessel occlusion with very low recanalization rates.

In a study reported by Kish et al, successful embolization was achieved in $75 \%$ of the cases of acute arterial bleed using NBCA. They concluded that NBCA was an effective method to achieve embolization in acute arterial hemorrhage and was able to achieve hemostasis even in cases of failed coil or particulate embolization. ${ }^{14}$

Gelfoam has also been described in the treatment of renal bleed; however, Gelfoam provides temporary embolization for few weeks and this is not desirable in cases of renal hemorrhages. Second, nontarget embolization has been 
reported with Gelfoam. ${ }^{15}$ Fisher et al found that $20 \%$ cases had loss of 30 to $50 \%$ renal function due to nontarget embolization in cases of renal artery pseudoaneurysm embolization. ${ }^{16}$ Gelfoam also has an added disadvantage since it leads to an increased risk of rupture of the pseudoaneurysm due to increased pressure while injection. ${ }^{17}$

The most common renal arterial injury observed in literature has been pseudoaneurysm followed by AVF with a rare occurrence of both of these entities together. ${ }^{18}$ The lifethreatening renal AVFs are noted in less than $0.5 \%$ of the cases. ${ }^{19}$ Our study found the incidence of pseudoaneurysm to be highest accounting for $74.2 \%$ (23 patients) of the cases with one patient showing simultaneous presence of two pseudoaneurysms. Three patients showed combined occurrence of pseudoaneurysm and AVF.

In cases of pseudoaneurysm with active extravasation into the urinary tract, glue can be used as an embolic agent. In a study, Ząbkowski et al evaluated 20 patients with bleeding into the urinary tract after PCNL. These patients were treated with a mixture of NBCA and lipiodol, mostly in a ratio of 1:3. Only one of these had bleeding with AVF, in whom for improving the safety, 1:1 mixture was used. Technical success was achieved in 20 cases and clinical success was achieved in 19 cases. The only complication noted was inadvertent and asymptomatic migration of glue and lipiodol mixture in the pulmonary vasculature in the patient with AVF. ${ }^{20}$ In our study out of the 31 patients, we have reported four patients with active extravasation and one patient with pseudoaneurysm and active extravasation and we have achieved $100 \%$ technical and clinical success in all these five patients with a glue and lipiodol mixture in a ratio of $1: 1$.

Lipiodol is used as a preferred diluting agent along with NBCA. There are majorly two roles of lipiodol. First, it acts as a contrast agent since glue is radiolucent. Second, it affects the polymerization time of NBCA and hence the adjusting the dilution ratio can be used to achieve a more controlled glue embolization. More dilution with lipiodol prolongs the time of glue polymerization as well as helps to achieve a more distal embolization. ${ }^{21-23}$

The most common cause of renal injury has been found to be iatrogenic with the highest incidence being attributed to percutaneous renal biopsies in various studies ranging from 7.4 to $11 \% .^{24,25}$ This is followed by percutaneous nephrostomy and percutaneous nephrolithotomy. ${ }^{26}$ In our study, renal biopsy was found to be the major cause closely followed by percutaneous nephrolithotomy.

A mean renal parenchymal loss of around 15\% has been described in literature on immediate postembolization check conventional angiography. The study by Poulakis et al has reported a loss of parenchyma ranging from 0 to 20\% (mean: 9\%). ${ }^{27}$ Another study by Chatziioannou et al reported a mean parenchymal ischemia of $12 \%$ in immediate postprocedure contrast-enhanced $\mathrm{CT}^{3}$ All of the above studies employed various embolizing agents that included coils, NBCA, polyvinyl alcohol, and Gelfoam. In our study with exclusive use of NBCA for embolization, we report an immediate postprocedural area percentage deficit from a range of $4 \%$ to $15.7 \%$ with a mean of $7.65 \%$.
It has also been observed that in the subsequent follow-up period, a reduction in the infarcted area to almost $50 \%$ of the initial area is found, although this parameter has not been evaluated in our study. ${ }^{27}$ This observation has been attributed to the fact that although renal arteries are end arteries, there is presence of intrarenal collateral supply via perivascular plexuses of segmental, interlobar, and arcuate arteries that eventually shrink the initial area of ischemia. ${ }^{3}$

Another parameter that has been used by authors to evaluate that the efficacy of embolization is the postembolization change in creatinine value. ${ }^{28}$ Srivastava et al reported that in the 24 patients in their study who underwent embolization to manage bleeding complication due to PCNL, there was no significant creatinine change after the embolization. ${ }^{29}$ In another study, preprocedure creatinine was $1.6 \pm 1.4 \mathrm{mg} / \mathrm{dL}$ and postprocedure creatinine was $1.7 \pm 1.5 \mathrm{mg} / \mathrm{dL}$ with no significant changes in creatinine values $(p=0.202) .{ }^{30}$ Our study similarly reported no significant creatinine difference between the pre- and postprocedure period.

The technical and clinical success that has been observed in various studies available is between a range of 87 to $100 \%$ and 83.3 to $100 \%$, respectively. $2,20,28,31,32$ We too report a technical and clinical success rate of $100 \%$ with exclusive use of NBCA as an embolizing agent. Follow-up at 1 and 6 months showed no recurrence of bleed and hence retained 100\% clinical success.

The study has many limitations. The method of calculation of renal infarction area is not accurate since it is done on conventional angiography in two dimensions. It can be agreed that a CT volumetry will provide a much accurate measure, but it was not feasible at our institute. Second, estimated glomerular filtration rate is a better indicator to assess the effect of glue embolization on the renal functions; however, our study had employed serum creatinine as an indicator that is less sensitive. Third, the INR values of the coagulopathic patients have been found to be in the range of 1.5 to 2 . Patients with INR $>2$ have not been observed in this study, which correspond to severe coagulopathy and hence can be considered as a limitation to very conclusively state the efficacy of NBCA in coagulopathy. Finally, the limited sample size and the study being a retrospective observational one are a major limitation, and hence an randomized controlled trial comparing various embolic agents such as glue versus coil is likely to offer significant insights. In conclusion, this study reports our experience with NBCA embolization in iatrogenic renal hemorrhages with achievement of complete technical and clinical success even on 6 months follow-up. It also establishes NBCA as an excellent embolizing agent in patients with coagulopathies. Finally, we also conclude that with exclusive glue embolization, there is minimal renal parenchymal loss along with no significant changes in renal function.

Conflicts of Interest

There are no conflicts of interest.

Acknowledgement

None. 


\section{References}

1 Huppert PE, Duda SH, Erley CM, et al. Embolization of renal vascular lesions: clinical experience with microcoils and tracker catheters. Cardiovasc Intervent Radiol 1993;16(06):361-367

2 Mavili E, Dönmez H, Ozcan N, Sipahioğlu M, Demirtaş A Transarterial embolization for renal arterial bleeding. Diagn Interv Radiol 2009;15(02):143-147

3 Chatziioannou A, Brountzos E, Primetis E, et al. Effects of superselective embolization for renal vascular injuries on renal parenchyma and function. Eur J Vasc Endovasc Surg 2004;28(02): 201-206

4 Malloy PC, Grassi CJ, Kundu S, et al. Standards of Practice Committee with Cardiovascular and Interventional Radiological Society of Europe (CIRSE) Endorsement. Consensus guidelines for periprocedural management of coagulation status and hemostasis risk in percutaneous image-guided interventions. J Vasc Interv Radiol 2009;20(Suppl 7):S240-S249

5 Vignali C, Lonzi S, Bargellini I, et al. Vascular injuries after percutaneous renal procedures: treatment by transcatheter embolization. Eur Radiol 2004;14(04):723-729

6 Phadke RV, Sawlani V, Rastogi H, et al. Iatrogenic renal vascular injuries and their radiological management. Clin Radiol 1997;52 (02):119-123

7 Dotter CT, Goldman ML, Rösch J. Instant selective arterial occlusion with isobutyl 2-cyanoacrylate. Radiology 1975;114(01): 227-230

8 Yonemitsu T, Kawai N, Sato M, et al. Evaluation of transcatheter arterial embolization with gelatin sponge particles, microcoils, and n-butyl cyanoacrylate for acute arterial bleeding in a coagulopathic condition. J Vasc Interv Radiol 2009;20(09):1176-1187

$9 \mathrm{Kim}$ J, Shin JH, Yoon HK, et al. Transcatheter renal artery embolization with N-butyl cyanoacrylate. Acta Radiol 2012;53(04): 415-421

10 Frodsham A, Berkmen T, Ananian C, Fung A. Initial experience using N-butyl cyanoacrylate for embolization of lower gastrointestinal hemorrhage. J Vasc Interv Radiol 2009;20(10): 1312-1319

11 Park JH, Kim HC, Chung JW, Jae HJ, Park JH. Transcatheter arterial embolization of arterial esophageal bleeding with the use of $\mathrm{N}$ butyl cyanoacrylate. Korean J Radiol 2009;10(04):361-365

12 Pollak JS, White RI Jr. The use of cyanoacrylate adhesives in peripheral embolization. J Vasc Interv Radiol 2001;12(08): 907-913

13 Takeuchi Y, Morishita H, Sato Y, et al. Committee of Practice Guidelines of the Japanese Society of Interventional Radiology. Guidelines for the use of NBCA in vascular embolization devised by the Committee of Practice Guidelines of the Japanese Society of Interventional Radiology (CGJSIR), 2012 edition. Jpn J Radiol 2014;32(08):500-517

14 Kish JW, Katz MD, Marx MV, Harrell DS, Hanks SE. N-butyl cyanoacrylate embolization for control of acute arterial hemorrhage. J Vasc Interv Radiol 2004;15(07):689-695

15 Savastano S, Feltrin GP, Miotto D, Chiesura-Corona M. Renal aneurysm and arteriovenous fistula. Management with transcatheter embolization. Acta Radiol 1990;31(01):73-76
16 Fisher RG, Ben-Menachem Y, Whigham C. Stab wounds of the renal artery branches: angiographic diagnosis and treatment by embolization. AJR Am J Roentgenol 1989;152(06):1231-1235

17 Moreau JF, Merland JJ, Descamps JM. Post-biopsy false arterial aneurysm of a transplanted kidney: treatment by bucrylate transcatheter embolization. J Urol 1982;128(01):116-118

18 Bryk DJ, Zhao LC. Guideline of guidelines: a review of urological trauma guidelines. BJU Int 2016;117(02):226-234

19 Winkelmayer WC, Levin R, Avorn J. Chronic kidney disease as a risk factor for bleeding complications after coronary artery bypass surgery. Am J Kidney Dis 2003;41(01):84-89

20 Ząbkowski T, Piasecki P, Zieliński H, Wieczorek A, Brzozowski K, Zięcina P. Superselective renal artery embolization in the treatment of iatrogenic bleeding into the urinary tract. Med Sci Monit 2015;21:333-337

21 Jae HJ, Chung JW, Jung AY, Lee W, Park JH. Transcatheter arterial embolization of nonvariceal upper gastrointestinal bleeding with N-butyl cyanoacrylate. Korean J Radiol 2007;8(01):48-56

22 Izaki K, Yamaguchi M, Kawasaki R, Okada T, Sugimura K, Sugimoto K. N-butyl cyanoacrylate embolization for pseudoaneurysms complicating pancreatitis or pancreatectomy. J Vasc Interv Radiol 2011;22(03):302-308

23 Kim BS, Do HM, Razavi M. N-butyl cyanoacrylate glue embolization of splenic artery aneurysms. J Vasc Interv Radiol 2004;15(1 Pt 1)91-94

24 Meng $\mathrm{CH}$, Elkin M. Immediate angiographic manifestations of iatrogenic renal injury due to percutaneous needle biopsy. Radiology 1971;100(02):335-341

25 Stiles KP, Yuan CM, Chung EM, Lyon RD, Lane JD, Abbott KC. Renal biopsy in high-risk patients with medical diseases of the kidney. Am J Kidney Dis 2000;36(02):419-433

26 Burrel M, Barrufet M, Sebastia MC, et al. Diffuse renal cortical hemorrhage in the setting of subcapsular hematoma: diagnosis and treatment with embolization. J Vasc Interv Radiol 2017;28 (11):1557-1562.e1

27 Poulakis V, Ferakis N, Becht E, Deliveliotis C, Duex M. Treatment of renal-vascular injury by transcatheter embolization: immediate and long-term effects on renal function. J Endourol 2006;20(06): 405-409

28 Sam K, Gahide G, Soulez G, et al. Percutaneous embolization of iatrogenic arterial kidney injuries: safety, efficacy, and impact on blood pressure and renal function. J Vasc Interv Radiol 2011;22 (11):1563-1568

29 Srivastava A, Singh KJ, Suri A, et al. Vascular complications after percutaneous nephrolithotomy: are there any predictive factors? Urology 2005;66(01):38-40

30 Güneyli S, Gök M, Bozkaya H, et al. Endovascular management of iatrogenic renal arterial lesions and clinical outcomes. Diagn Interv Radiol 2015;21(03):229-234

31 Chiramel GK, Keshava SN, Moses V, Kekre N, Tamilarasi V, Devasia A. Clinical outcomes of endovascularly managed iatrogenic renal hemorrhages. Indian J Radiol Imaging 2015;25(04):380-390

32 Seif HMA, Hameed DA. Endovascular management of iatrogenic renal vascular injury: experience in 28 cases. Med J Cairo Univ 2011;79(01):177-184 